
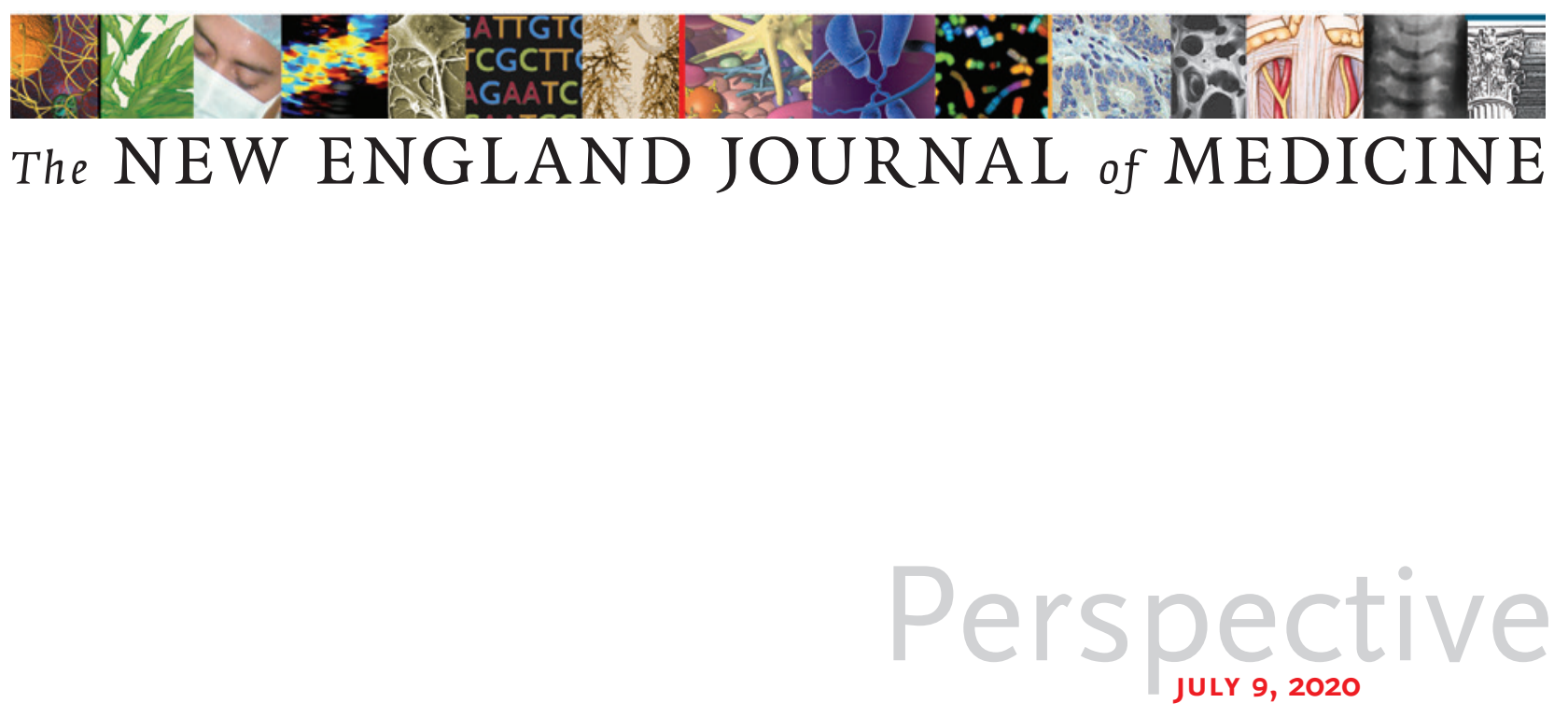

\title{
Social Prescribing - Transforming the Relationship between Physicians and Their Patients
}

Martin Roland, D.M., Sam Everington, M.B., B.S., and Martin Marshall, M.D.

\section{Mo many physicians who see patients with ill- nesses that are strongly influenced by social circumstances, socially oriented interventions make sense. The ideas behind such interventions}

are not new - community-oriented primary care in the United States has always had a strong focus on social rather than medical interventions, and the Centers for Medicare and Medicaid Services' Accountable Health Communities model addresses the health-related social needs of Medicare and Medicaid beneficiaries with a goal of reducing health care costs and utilization. The United Kingdom is now aiming to make similar nonmedical interventions mainstream. As part of a National Health Service (NHS) program, all primary care physicians in England will have access to a "link worker" to support so-called social prescribing, and an organization is being established to guide the training of such workers, mon- itor the availability of social-prescribing services on the ground, and ensure that these services are evaluated. ${ }^{1}$ The NHS plans to recruit 1000 social-prescribing link workers over the next 2 years and additional workers in subsequent years. The goal is for nearly 1 million patients to have been referred for social-prescribing interventions by 2024 .

Considerable claims have been made regarding the benefits of social prescribing, including that it reduces utilization of primary and secondary care, reduces rates of emergency department visits, and improves physical and mental health outcomes. Although more research is needed to substantiate these claims, we believe that social prescribing has the potential to address the fixation by both physicians and patients on tests and prescriptions that may bear little relation to the cause of a patient's problem.

Many patients present to their primary care physician with a concern that is either nonmedical or strongly related to the patient's socioeconomic circumstances. Even when there is a clear diagnosis, the most effective interventions may not be medical ones. Nevertheless, physicians are drawn to medical interventions, including prescribing drugs recommended by the plethora of disease-specific guidelines that have been developed over the past 20 years. Doctors are increasingly being criticized, however, for "overmedicalizing" health problems - a tendency that has led to a growing number of hospital admissions related to adverse effects of medications. The opioid crisis is perhaps the most striking example of overmedicalization. 
Such critiques lie at the heart of the Choosing Wisely and Too Much Medicine campaigns in the United States and United Kingdom, respectively, which seek to promote conversations between patients and clinicians that will help reduce the number of unnecessary tests, procedures, and prescriptions.

Alternative approaches recognize that lifestyle changes could help alleviate many problems and that there are frequently resources available in the community, many of which will encourage changes in health behaviors that can help patients manage their conditions. Physicians are often unaware of these resources, however, or don't know how to connect patients with them. The concept of social prescribing entails educating physicians about social interventions, providing guidance on local resources, and permitting them to "prescribe" social interventions for patients. Social interventions in the United Kingdom may be funded by charities, private business, or, on occasion, by the NHS or local authorities. Socialprescribing programs have generally focused on elderly people, people with mental health problems, and those living in socioeconomically deprived communities.

Some of these interventions have a clear biomedical intent for example, exercise and weightreduction programs to reduce dependence on medication among people with diabetes (see box). But social prescribing has a wider purpose. It's also about culture change - challenging the propensity to medicalize health and professionalize health care. And it's about changing the expectations of patients (and their physicians) that a drug will solve their problems by empowering

\section{Examples of Social Interventions.}

Gym or exercise classes or groups

Weight-management and nutrition interventions

Art-based therapies

Employment or volunteer opportunities

Self-help groups for specific conditions

Parenting programs

Advice services (e.g., for welfare entitlements, housing, or debt)

Community activities, including gardening, cooking, sports, and befriending

patients to invest in their own health. Patients from poor communities and those with low health literacy may particularly benefit from improved access to community resources.

There are many types of social interventions, and outcomes measured in assessments of social prescribing range widely, from health care utilization and healthrelated quality of life to patient and physician satisfaction. Nevertheless, published evaluations have identified some common outcomes of these interventions. Perhaps most striking are the strongly positive qualitative evaluations given by patients and physicians. Claims of benefits in terms of quantitative outcomes (such as health care utilization) are harder to evaluate because many studies are small, lack controls, and are of poor quality. Although systematic reviews have found that many published studies of social interventions report some positive outcomes, the poor quality of such studies led one group of reviewers to conclude that "current evidence fails to provide sufficient detail to judge either success or value for money.",2,3

We believe that the NHS should take advantage of its large-scale experiment in social prescribing to carry out research on which patients are most likely to benefit from social interventions and which interventions are most likely to be effective for both physical and mental health problems. It will also be important to learn how best to avoid the risk of social prescribing's becoming a "tickbox" exercise, rather than an approach that genuinely engages with a patient's needs, and to elucidate the role of social-prescribing link workers in supporting these interventions. ${ }^{4}$ Payers will want to know the costs and benefits of such programs, and evaluations also need to consider the possible adverse effects of social interventions that might divert patients from more effective interventions. Some programs may be ineffective simply because of lack of community resources. As one commentator put it, "Just as a doctor's prescription can only improve health if the patient has access to a well stocked pharmacy, so social prescribing schemes depend on a well stocked community." 5

Questions have been posed about whether social prescribing is an appropriate activity for physicians, many of whom already have unmanageable workloads, and whether it runs the risk of further medicalizing problems whose solutions should be outside the sphere of medicine. Our view is that social prescribing is appropriate and important. All physicians should have learned about social determinants of health and biopsychosocial models of illness during medical school. Such education, however, seems to have had little effect on their propensity to reach for the prescription pad to address problems that are not fundamentally amenable to medical therapy.

Patients will continue to pre- 
sent with problems that are purely medical, purely social, or (for a large group in the middle) caused by a mixture of medical and social factors. The aim of social prescribing is to give physicians access to interventions that should reduce unnecessary prescriptions and referrals and encourage patients to take responsibility for An audio interview their health by givwith Dr. Roland is available at NEJM.org ing them the capacity and opportunity to use their personal resources and those in their families and communities. Social interventions could also reduce disparities in health - particularly interventions that focus on socially disadvantaged communities where patients' medical problems are especially likely to be compounded by social difficulties.

Social prescribing has the potential to change the consultation in ways that have profound implications for medical practice and medical education, but physicians need reliable information on what interventions work best and for whom and how social prescriptions can best be integrated into conventional medical practice.

Disclosure forms provided by the authors are available at NEJM.org.

From the University of Cambridge, Cambridge (M.R.), and the Bromley by Bow Health Centre (S.E.) and the Royal College of General Practitioners (M.M.), London all in the United Kingdom.

1. NHS England. Social prescribing and community-based support: summary guide. January 31, 2019 (https://www.england.nhs .uk/wp-content/uploads/2019/01/social -prescribing-community-based-support -summary-guide.pdf).

2. Bickerdike L, Booth A, Wilson PM, Farley K, Wright K. Social prescribing: less rhetoric and more reality - a systematic review of the evidence. BMJ Open 2017;7(4): e013384.

3. Chatterjee HJ, Camic PM, Lockyer BL, Thomson LJM. Non-clinical community interventions: a systematised review of social prescribing schemes. Arts Health 2018;10: 97-123.

4. Moffatt S, Steer M, Lawson S, Penn L, O'Brien N. Link Worker social prescribing to improve health and well-being for people with long-term conditions: qualitative study of service user perceptions. BMJ Open 2017; 7(7):e015203.

5. McNally L. Tackling loneliness with a well stocked community. BMJ 2018;362: k2953.

DOI: 10.1056/NEJMp1917060

Copyright (C) 2020 Massachusetts Medical Society.

\section{Ensuring Choice for People with Kidney Failure - Dialysis, Supportive Care, and Hope}

Kamyar Kalantar-Zadeh, M.D., M.P.H., Ph.D., Aaron Wightman, M.D., and Solomon Liao, M.D.

$\mathrm{B}$ ach year in the United States, approximately 120,000 people with kidney failure choose to start dialysis therapy - an arduous, life-sustaining treatment and more than half a million patients already receiving dialysis continue their treatment. More than $80 \%$ of these patients receive hemodialysis therapy three times per week in an outpatient dialysis clinic, which involves frequent travel that can be particularly challenging for elderly people and frail patients with unstable conditions. Because of the lack of kidneys available for transplantation, less than $5 \%$ of patients who have been receiving dialysis undergo transplantation each year. ${ }^{1}$

In a July 2019 executive order, President Donald Trump called for increased utilization of home dialysis and kidney transplantation; moreover, the Department of Health and Human Services hopes to achieve a $25 \%$ reduction in the incidence of end-stage kidney disease by 2030. Many potentially beneficial changes could come out of this executive order, including the expansion of conservative and preservative management of chronic kidney disease (CKD) without dialysis and more effective symptom management (see diagram). There is also an emerging perception among physicians and other experts that palliative and supportive care without renal replacement therapy may be increasingly leveraged for many current and prospective dialysis recipients.
Dialysis treatment prolongs life, but it can be burdensome for patients and their care partners. It has physical, psychosocial, and financial impacts similar to those of chemotherapy for advanced cancer, and patients starting dialysis will live an average of less than 5 years more. Given these burdens, palliative medicine specialists may be engaged in providing guidance for current and prospective dialysis recipients and their caregivers. Supportive care can address symptoms such as pain, fatigue, anxiety, and depression and can promote understanding of prognosis, discussions about goals of care, advance care planning, and consideration of compassionate, conservative care.

However, involvement of pal- 